\title{
Mild cognitive disorders are associated with different patterns of brain asymmetry than normal aging: the PATH through life study
}

\author{
Nicolas Cherbuin ${ }^{1}$, Chantal Réglade-Meslin ${ }^{1}$, Rajeev Kumar ${ }^{1,2}$, Perminder Sachdev ${ }^{3,4}$ and Kaarin J. Anstey \\ 1 Centre for Mental Health Research, Australian National University, Canberra, ACT, Australia \\ 2 Department of Psychological Medicine, Australian National University, Canberra, ACT, Australia \\ ${ }^{3}$ School of Psychiatry, University of New South Wales, Sydney, NSW, Australia \\ ${ }^{4}$ Neuropsychiatric Institute, Prince of Wales Hospital, Sydney, NSW, Australia
}

\section{Edited by:}

Marc Dhenain, CEA, France

Reviewed by:

Olivier Colliot,

Centre National de la Recherche

Scientifique, France

Judy Pa, University of California, USA

*Correspondence:

Nicolas Cherbuin, Centre for Mental Health Research, 63 Eggleston Road, Australian National University,

Canberra, ACT 0200, Australia.

e-mail:nicolas.cherbuin@anu.edu.au

Statistical analysis: Nicolas Cherbuin ${ }^{1}$
Background and Purpose: Defining how brain structures differ in pre-clinical dementia is important to better understand the pathological processes involved and to inform clinical practice. The aim of this study was to identify significant brain correlates (volume and asymmetry in volume) of mild cognitive disorders when compared to normal controls in a large community-based sample of young-old individuals who were assessed for cognitive impairment. Methods: Cortical and sub-cortical volumes were measured using a semi-automated method in 398 participants aged 64-70 years who were selected from a larger randomly sampled cohort and who agreed to undergo an MRI scan. Diagnoses were reached based on established protocols for $\mathrm{MCl}$ and a more inclusive category of any Mild Cognitive Disorder (any-MCD: which includes AAMI, AACD, $\mathrm{OCD}, \mathrm{MNC}, \mathrm{CDR}, \mathrm{MCl})$. Logistic regression analyses were used to assess the relationship between volume and asymmetry of theoretically relevant cerebral structures (predictors) and $\mathrm{MCl}$ or any-MCD while controlling for age, sex, and intra-cranial volume. Results: The main correlates of cognitive impairment assessed in multivariate analyses were hippocampal asymmetry (more to left, $\mathrm{MCl}$ : OR 0.83, 95\% Cl 0.71-0.96, $p=0.013$; MCD: OR 0.86, 95\% Cl 0.77-0.97, $p=0.011$ ), lateral ventricle asymmetry (more to left, $\mathrm{MCl}$ : OR $0.95,95 \% \mathrm{Cl} 0.91-0.99, p=0.009 ; \mathrm{MCD}$ : OR $0.95,95 \% \mathrm{Cl} 0.92-0.98, p=0.004$ ), and cerebellar cortex asymmetry (more to right, $\mathrm{MCl}$ : OR 1.51, 95\% $\mathrm{Cl} 1.13-2.01, p=0.005)$. Conclusions: In this population-based cohort stronger associations were found between asymmetry measures, rather than raw volumes in cerebral structures, and mild cognitive disorders.

Keywords: mild cognitive impairment, MRI, hippocampus, ventricle, cerebellum

\section{INTRODUCTION}

Previous studies have demonstrated an association between MCI diagnosis (and other mild cognitive disorders) and cerebral atrophy, particularly in the hippocampus, amygdala, and entorhinal cortex (see Anstey and Maller, 2003 for a review) as well as in other cortical areas, with high atrophy rates detected in temporo-parietal regions (Desikan et al., 2008). However, other studies have found no such association. For example, a study using a sub-sample of that assessed in the present investigation and based on a previous wave of measurement in 60- to 64-year olds found no association between a number of manual and automated brain measures and MCI status (Kumar et al., 2006). These findings raise the possibility that structural brain changes associated with MCI are not yet easily detectable in the young-old or that structural changes other than regional volumes are indicative of MCI related neuropathology.

Previous findings have suggested that variation in the relationship between left and right volumes of structures such as the hippocampus might be associated with neurodegenerative processes specifically involved in mild cognitive disorders and dementia. Thompson et al. (2007) showed that in Alzheimer's disease (AD) cortical atrophy occurred earlier and progressed faster in the left than in the right hemisphere. In other studies, hippocampal asymmetry was found to be reduced in subjects suffering from dementia (Barnes et al., 2005), specific regional hippocampal atrophy patterns were found in MCI and particularly in the left hippocampus (Apostolova et al., 2010), and in a meta-analysis Shi et al. (2009) demonstrated that left hippocampi were smaller than right hippocampi in MCI and AD. Moreover, ventricular asymmetry has been shown to be associated with metabolic asymmetry in frontotemporal dementia (Jeong et al., 2005), asymmetry in cortical metabolic activity in $\mathrm{AD}$ patients was correlated with cerebellar and basal ganglia metabolic asymmetry indexes (Akiyama et al., 1989), and amygdalar asymmetry discriminated between Alzheimer's disease and fronto-temporal lobar degeneration (Barnes et al., 2006).

Recently, Giannakopoulos et al. (2009) investigated the relationship between the asymmetrical distribution of Alzheimer's disease (amyloid plaques and neurofibrillary tangles) and microvascular pathology in the left and right hemispheres and cognition in 153 individuals whose brain was examined in a post-mortem study. They found that while Alzheimer's disease pathology was not asymmetrically distributed cerebral microvascular pathology was, with many more brains presenting with a predominance 
of microvascular pathology in the left hemisphere. In addition, cognitive status assessed with the CDR scale was positively associated with Alzheimer and microvascular pathology in each hemisphere. Together these findings suggest that atypical cerebral asymmetry as well as atrophy might be associated with MCI and dementia.

In the present study, we aimed to identify the brain correlates of MCI and other related mild cognitive disorders in comparison to normal controls in a large sample of community-dwelling individuals aged 64-70 participating in the second wave of assessment of a longitudinal investigation of aging and mental disorders: the PATH through life study. Because MCI status is relatively unstable and because we showed in a previous study (Anstey et al., 2008a) that a somewhat less specific and overlapping clinical group including any mild cognitive disorder (any-MCD: composed of AAMI, AACD, OCD, MNC, CDR, MCI) was very stable ( $89 \%$ over 4 years) we also investigated the cerebral correlates of this sub-group.

In order to investigate the association between cerebral asymmetry as well as raw cerebral volumes and cognitive impairment in the present study we computed an asymmetry index for each lateralized structure measured and included these indexes in all analyses. It was expected that MCI and any-MCD would be associated with greater cerebral atrophy, particularly in medial temporal structures, ventricular expansion, and with reduced hippocampal asymmetry (i.e. relatively smaller left than right volumes in cognitively impaired participants). The relationship between the asymmetry of other structures and cognitive status was considered exploratory.

\section{MATERIALS AND METHODS STUDY POPULATION}

The design of the PATH through life study has been described elsewhere (Jorm et al., 2004) as has the clinical sub-study (Kumar et al., 2005). Briefly, participants who were residents of the city of Canberra and the adjacent town of Queanbeyan, Australia, were recruited randomly through the electoral roll to participate in a study interested in the risk and protective factors for normal aging, dementia and other neuropsychiatric disorders. Enrolment to vote is compulsory for Australian citizens. Participants were recruited in three age cohorts 20-24, 40-44, 60-64 and are to be followed every 4 years, over a total period of 20 years. The study was approved by the Australian National University Ethics Committee. Results presented here concern the second-wave interviews with 2222 participants aged 64-70 years which were conducted in 2005-2006. Of these, 421 individuals underwent an MRI scan (18.9\%) and 417 completed the full cognitive assessment and were included in the present study (4 refusals).

\section{CLINICAL ASSESSMENT}

The screening procedures and clinical diagnosis used in the PATH study have been described previously (Anstey et al., 2008b). In brief, diagnoses were obtained for MCI (Petersen et al., 1999), Age Associated Memory Impairment (AAMI) (Kral, 1962), Age Associated Cognitive Decline (AACD) (Crook et al., 1986), Mild Neurocognitive Disorder (MNC) (American Psychiatric Association, 1994), Impairment on the Clinical Dementia Rating (CDR) scale (Morris, 1993), and Other Cognitive Disorder (OCD) using published criteria (Kumar et al., 2006). DSM-IV criteria were used to assess dementia (American Psychiatric Association, 1994).
While a focus of this research is MCI, it has become evident that this clinical category is not very stable (Anstey et al., 2008a) with many individuals given an MCI diagnosis at one time point moving across to other mild cognitive disorder categories at follow-up. Since we have previously reported high stability (89\%) of an overlapping and more inclusive diagnosis of any mild cognitive disorder (defined by any of MNC, OCD, AAMI, MCI, AACD, CDR) over a 4 year follow-up (Anstey et al., 2008a), participants were also classified with any-Mild Cognitive Disorder (any-MCD) and analyses were conducted to identify brain correlates of this more general classification.

\section{MRI SCAN ACQUISITION}

All participants were imaged with a 1.5 Tesla Philips Gyroscan ACS-NT scanner (Philips Medical Systems, Best, The Netherlands) for T1-weighted 3-D structural MRI. The T1-weighted MRI was acquired in coronal orientation using a T1-FFE sequence with the following parameters: $\mathrm{TR}=8.93 \mathrm{~ms}, \mathrm{TE}=3.57 \mathrm{~ms}$, flip angle of $8^{\circ}$, matrix size $=256 \times 256$, slices 160, and field of view (FOV) $256 \times 256 \mathrm{~mm}$. Slices were contiguous with slice thickness of $1.5 \mathrm{~mm}$.

\section{IMAGE ANALYSIS}

Volumetric segmentation was performed with the Freesurfer image analysis suite, which is documented and freely available for download online (http://surfer.nmr.mgh.harvard.edu/). This processing includes motion correction, removal of non-brain tissue using a hybrid watershed/surface deformation procedure (Segonne et al., 2004), automated Talairach transformation, and segmentation of the sub-cortical white matter and deep gray matter volumetric structures (Fischl et al., 2002, 2004). For the purpose of the present study only a subset of relevant segmented structures were selected for analysis and included the hippocampus, amygdala, thalamus, caudate, putamen, pallidum, ventricles, cerebellum, and total gray and white matter for each hemisphere (see Figure 1). In addition, to assess the role of differences in left and right brain structures an asymmetry index was computed for each left-right pairs using the equation: $[(\mathrm{L}-\mathrm{R}) /(\mathrm{L}+\mathrm{R})] \times 100$ which produces an index ranging from -100 (larger right volume) to +100 (larger left volume). The scans of nineteen participants were excluded from the sample due to poor scan quality, low signal-to-noise ratio, or movement artefacts which did not allow for normal processing with the standard Freesurfer pipeline leaving 398 participants for analysis. Each segmented volume was inspected slice by slice and reprocessed with additional parameters if errors were detected.

\section{STATISTICAL ANALYSIS}

Descriptive analyses were conducted using Chi-square for categorical data and t-tests to compare groups on continuous variables. Logistic regression analysis was used to identify significant predictors of binary group membership (normal vs. MCI or normal vs. any-MCD) using a stepwise approach. In a first phase, each brain variable was assessed in a univariate analysis while controlling for age, sex, and intracranial volume. In a second phase, multivariate logistic regression analyses were conducted to determine which variables had the highest predictive value while controlling for age, sex, and intracranial volume. All brain variables used as predictors in logistic regression analysis were centered and scaled so each 
unit represented a $1 \mathrm{ml}$ volume deviation from the mean. To avoid singularities left brain and asymmetry measures were used in multivariate analyses and checked against the same analyses using the right brain measures. The alpha level was set at $p=0.05$.

\section{RESULTS}

Table 1 presents the demographic and neuropsychological characteristics and Table 2 the brain measures of the study groups (Normal, MCI, and any-MCD). Twenty-two MCI, 37 any-MCD
(22 MCI, 10 AAMI, 19 AACD, 2 MNC, 2 OCD; diagnostic categories are not mutually exclusive) and 361 control individuals were identified from the 398 participants who were included in the study and whose imaging data could be used for analysis. No case of dementia was detected. The MCI and any-MCD groups did not differ from the normal group based on age, but they included significantly fewer females, had fewer years of education, a greater proportion of participants from a non-caucasian and non-English-speaking background. The three groups did

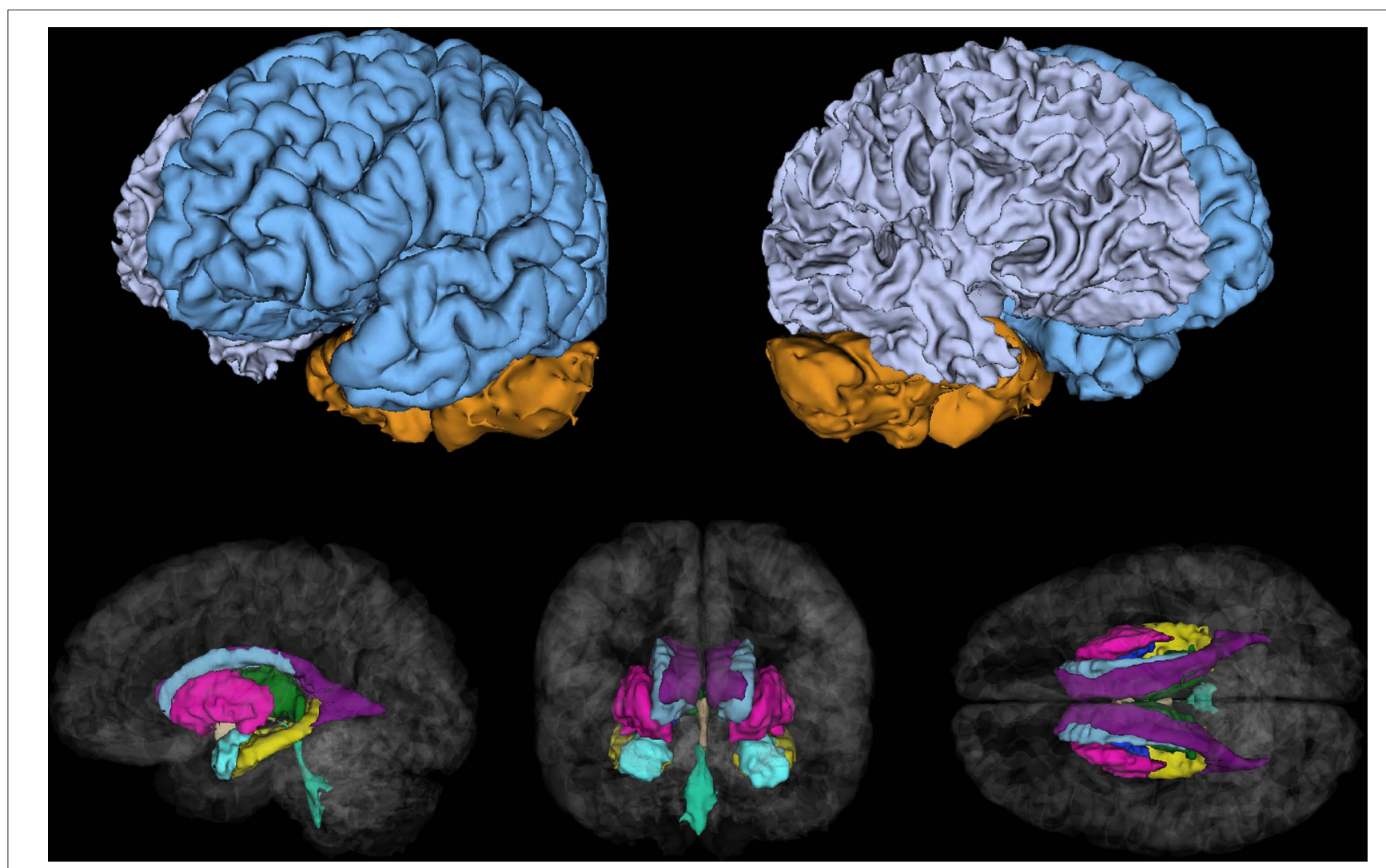

FIGURE 1 | Cerebral structures segmented in Freesurfer and investigated in the present study including gray/white matter and cerebellum (top), caudate (gray), pallidum (dark blue), thalamus (green), putamen (pink), hippocampus (yellow), amygdala (light blue), and lateral (purple), 3rd (beige) and 4th (turquoise) ventricles (bottom).

Table 1 | Demographic and Neuropsychological characteristics of the normal, MCl, and any-MCD sub-samples.

\begin{tabular}{lccc}
\hline Demographic variables & Normal $(\boldsymbol{n = 3 6 1 )}$ & MCI $(\boldsymbol{n = 2 2 )}$ & Any-MCD (n= 37) \\
\hline Female (\%, SD) & $172(47.7)$ & $5(22.7)^{*}$ & $10(27.0)^{*}$ \\
Age at wave 1 (years, SD) & $66.58(1.44)$ & $66.18(1.56)$ & $66.14(1.55)$ \\
Education (years, SD) & $14.19(2.68)$ & $12.39(3.14)^{* *}$ & $17(77.27)^{* *}$ \\
Caucasian (\%, SD) & $345(95.60)$ & $11(50.00)^{* *}$ & $31(83.80)^{* *}$ \\
English-speaking (\%, SD) & $330(91.90)$ & $27.05(2.65)^{* *}$ & $23(62.16)^{* *}$ \\
MMSE (SD) & $29.39(1.03)$ & $4.05(1.94)^{* *}$ & $27.50(2.30)^{* *}$ \\
Immediate recall (SD) & $7.24(1.95)$ & $2.77(2.31)^{* *}$ & $4.30(1.76)^{* *}$ \\
Delayed recall (SD) & $6.36(2.28)$ & $39.32(13.29)^{* *}$ & $3.14(1.96)^{* *}$ \\
Symbol-digit modalities test (SD) & $50.82(8.30)$ & $40.81(12.34)^{* *}$ \\
\hline
\end{tabular}

*Significant at $p<0.05,{ }^{*}$ Significant at $p<0.001$. 
Table 2 | Brain measures of the normal, $\mathrm{MCl}$, and any-MCD sub-samples.

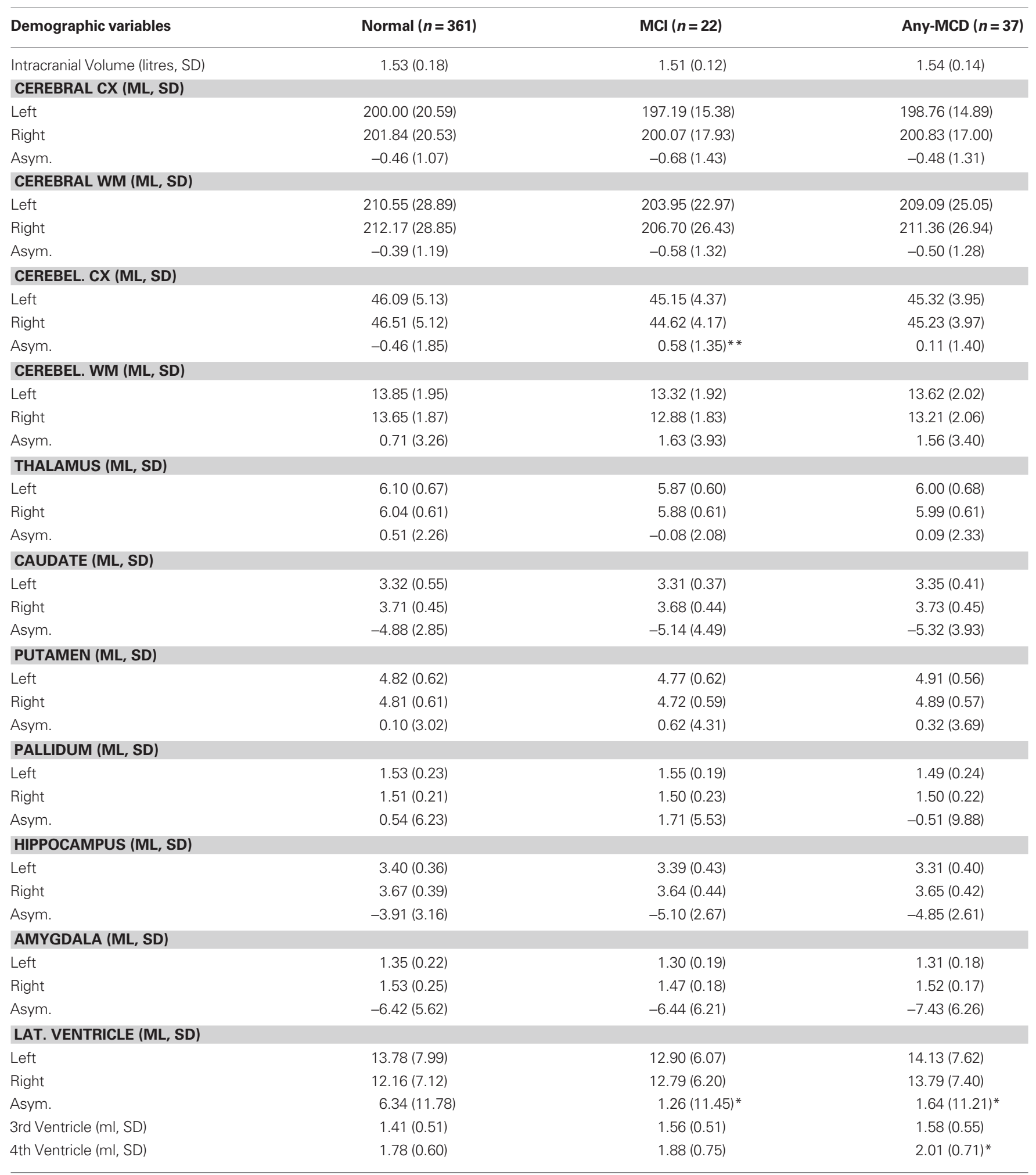

${ }^{*}$ Significant at $p<0.05,{ }^{*}$ Significant at $p<0.001 ; C X=$ cortex, $W M=$ white matter, Cerebell. $=$ cerebellum, Asym. = Asymmetry Index.

not differ on any of the raw brain variables except for the 4 th ventricle which was smaller in the normal compared to the anyMCD group.

\section{MCI/ANY-MCD BRAIN CORRELATES}

Results of the logistic regressions assessing the brain correlate of $\mathrm{MCI} /$ any-MCD by univariate analysis while controlling for age, sex, 
and intra-cranial volume are presented in Table 3 and show that right cerebellar volume, cerebellar asymmetry, left thalamic volume, left hippocampal volume, left amygdalar volume, and lateral ventricle asymmetry were significant predictors.
In a second step, all brain variables investigated in univariate analyses (left hemisphere volume and asymmetry measures; the variance of right hemisphere volumes is indexed in the combination of left hemisphere measures and asymmetry indexes) were

Table 3 | Univariate correlates of $\mathrm{MCl}$ and any-MCD after controlling for sex, age, and intra-cranial volume.

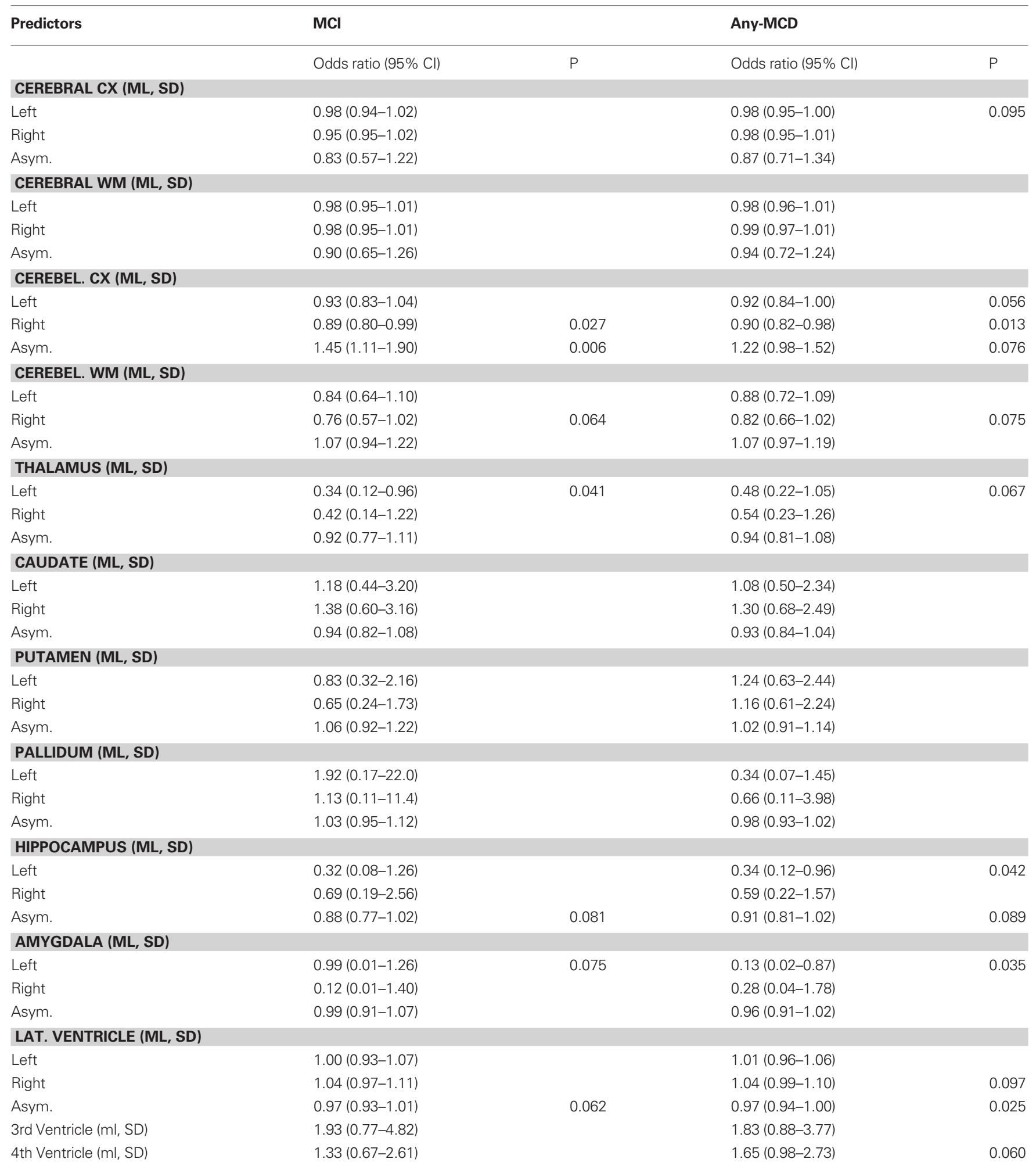


entered concurrently in multivariate analyses while controlling for age, sex, and intra-cranial volume and after reduction of the model, the significant predictors were the following: cerebellar cortex asymmetry (MCI: OR 1.51,95\%CI 1.13-2.01, $p=0.005$ ), lateral ventricle asymmetry (MCI: OR 0.95, 95\%CI 0.91-0.99, $p=0.009$; MCD: OR 0.95, 95\%CI 0.92-0.98, $p=0.004)$, and hippocampal asymmetry (MCI: OR 0.83, 95\%CI 0.71-0.96, $p=0.013$; MCD: OR $0.86,95 \%$ CI $0.77-0.97, p=0.011$ ). None of the absolute volume measures remained in the model after reduction beside the asymmetry measures described above. The reduced model fit was significantly improved by the introduction of the brain variables: MCI, $-2 \log$ likelihood $=139.45$ vs 157.12, Cox and Snell $R^{2}=0.073$; any-MCD, $-2 \log$ likelihood $=224.25$ vs 236.04, Cox and Snell $R^{2}=0.025$. The same analyses were repeated using right hemisphere and asymmetry measures and showed identical results. In addition, because there were small but significant differences in education levels and ethnicity between the clinical groups and controls we repeated all analyses with education and ethnicity as covariate. No substantial difference were evident and results followed the same pattern of results. Figure 2 shows the relationship between left and right volume differences and asymmetry indexes for the hippocampus, lateral ventricle, and cerebellum.

\section{DISCUSSION}

The present results show that while right cerebellar volume, left thalamic volume, left hippocampal volume, and left amygdalar volume were significant correlates of MCI and/or any-MCD, most raw volume measures were not associated with cognitive decline before or after controlling for relevant covariates. These findings appear to indicate that the early signs of clinical impairment in aging are not associated with large volumetric differences in most cerebral structures but mainly co-occur with left medial temporal lobe change.

In contrast, the strongest correlates of cognitive status in the present study were measures of asymmetry of the hippocampus, cerebellum, and lateral ventricle. As in previous studies it was found that the right hippocampus was larger than the left in a majority of participants and this trend was slightly stronger in cognitively normal individuals. However, a somewhat counter-intuitive result was also found. Contrary to a number of studies reporting on asymmetries of structures in cognitive impairment and AD which defined asymmetry by a simple difference between left and right volumes we computed an index widely used in the laterality literature which normalized for total structure volume and which has a fixed range $(-100$ to +100$)$. With this index it was found that larger left hippocampal asymmetry was associated with decreased odds of cognitive impairment which appears to conflict with previous findings but does not. This relationship can be better understood in light of the fact that the asymmetry index is influenced by the direction and the strength of the difference between left and right structures and their normalization in controlling for other factors (age, sex, education, hippocampal and intra-cranial volume; see Figure 1). Therefore although cognitively normal participants had on average larger left and right raw volumes than cognitively

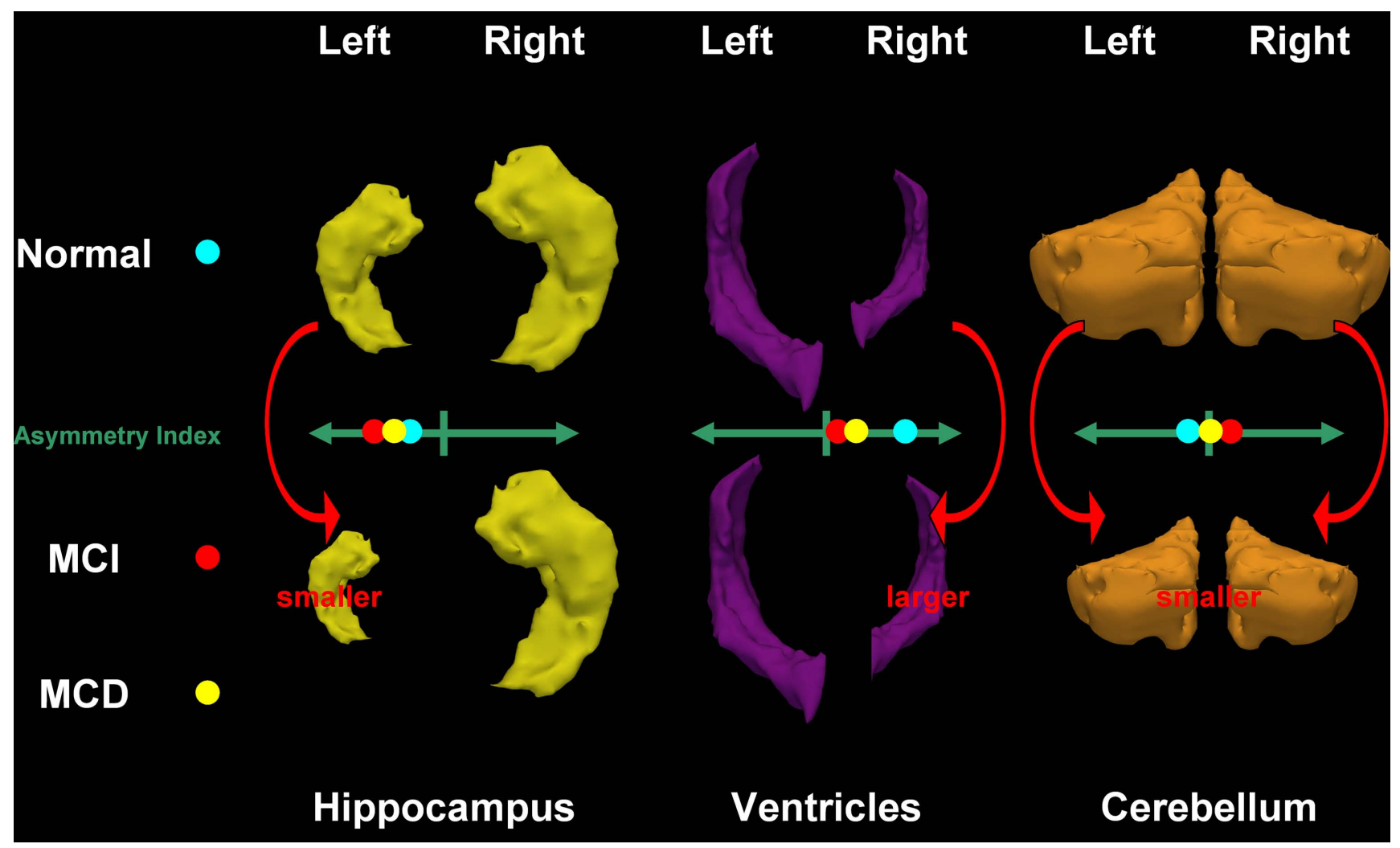

FIGURE 2 | Hippocampus, ventricle, and cerebellum models showing relative differences in left and right structures and the direction of the associated asymmetry indexes in cognitively normal participants and in individuals with $\mathrm{MCl}$. 
impaired participants with the right hippocampus being larger than the left hippocampus, their asymmetry index indicated that an overall larger relative left asymmetry, or more precisely a lesser right asymmetry, was associated with decreased odds of cognitive impairment. This finding might indicate that change in relative size of the left and right hippocampi is more important than differences in absolute size in each of these structures. Furthermore, at least in this cohort, this variation in symmetry appears to be more strongly influenced by the left hippocampus with a larger size being associated with significant decreased odds of cognitive impairment whereas right hippocampal size was not associated with increased or decreased odds of cognitive impairment. This interpretation is congruent with other results in the present study showing that apart from the putamen, pallidum, and cerebellum, greater left or lesser right asymmetry in all other gray and white matter structures was associated with decreased odds of presenting with cognitive impairments (and vice versa for the lateral ventricles). Or in other words, individuals with proportionally smaller left hemisphere volumes (rather than larger right hemisphere volumes) were more likely to be diagnosed with MCI or any-MCD which is consistent with data reported by Thompson et al. (2007).

It is interesting to note that the opposite relationship found in the cerebellum (in this study) appears to mirror that described in a metabolic study where decreased activity in the left basal ganglia of $\mathrm{AD}$ patients was found to be correlated with decreased activity in the right cerebellum (Akiyama et al., 1989), and is suggestive of a contra-lateral association between basal ganglia and cerebellum.

Based on the previous results it might have been expected that the greater risk of impairment associated with relatively smaller left than right hippocampi would also have been associated with relatively larger left than right lateral ventricles. Instead we found the opposite, while cognitively impaired participants had smaller left ventricles than cognitively normal individuals, a smaller asymmetry index (driven by a larger right ventricle) was associated with a greater risk (see Figure 1). Giannakopoulos et al. (2009) found in a post-mortem study that although microvascular pathology was most prevalent in the left hemisphere and correlated with cognitive impairment, the conjunction of Alzheimer and microvascular pathology in the right hemisphere was most predictive of dementia. Thus the present finding could suggest that when significantly increased right ventricular expansion becomes apparent it is indicative of the compounding effects of Alzheimer and microvascular pathology in the right hemisphere which is likely to occur later in the disease progression and to be associated with an inability of the right hemisphere to compensate for the functional impairment associated with earlier progression of pathological processes in the left hemisphere. The ventricles may be particularly sensitive indicators of this phenomenon since periventricular and deep lesions could exercise a greater influence on ventricular volume due to their localization (Wen et al., 2006), and other factors which could influence ventricular volume, such as total cerebral white matter atrophy, may well be more reflective of other less relevant influences such as sulcal widening and sub-cortical white matter lesions. In addition, periventricular lesions and deep white matter lesions have been shown to be particularly predictive of impairment in aging (van den Heuvel et al., 2006; Yoshita et al., 2006; Oosterman et al., 2008).
Cerebral asymmetry in anatomy and function has been extensively described in the literature. However, the origin of asymmetrical changes/differences in neuropathological processes associated with aging and dementia remains a mystery. It has been suggested that the left hemisphere is more sensitive to stress with a recent meta-analysis of hippocampal volumes in PTSD confirming greater left than right atrophy in this clinical group (Smith, 2005). It is also possible that these asymmetry differences are due to chronic hypoperfusion of the left hemisphere. In a SPECT study of 300 outpatients, it was found that women were more likely than men to present with significantly decreased perfusion in the left compared to the right hemisphere and that this pattern was more marked in those identified as having probable AD (Ott et al., 2000). Consistent findings were also found in another study comparing $18 \mathrm{AD}$ patients with 20 controls (Trollor et al., 2005). Moreover, recent research has showed that common carotid artery intima-media thickness correlated positively with age and was greater on the left side and particularly so in left-handed subjects (Onbas et al., 2007) suggesting that hemodynamic stress and intimal damage could be larger in the left carotid artery. Consistent with this hypothesis Giannakopoulos et al. (2009) found that microvascular pathology, but not Alzheimer pathology, was more prevalent in the left than in the right hemisphere of 153 brains of individuals with different levels of impairment and who were assessed in a post-mortem study. In contrast, Amyloid Beta deposits assessed with Pittsburgh compound B imaging in MCI and AD showed no asymmetry (Raji et al., 2008; Giannakopoulos et al., 2009). Taken together these results seem to indicate that difference in vulnerability between left and right cerebral structures in aging are more likely to be associated with cardio-vascular risk factors which in turn accentuate neurodegenerative processes prodromal to cognitive impairment and dementia.

The present results are of particular interest because a previous study conducted on a sub-sample of the same study as this one found no cerebral correlates of MCI in the first wave of measurement conducted 4 years earlier. The differences found between cognitively normal individuals and those diagnosed with MCI are therefore likely to have developed over the last 4 years. It is also interesting to note that the cerebral correlates of MCI and any-MCD were very similar and suggest a continuum or large overlap between different mild cognitive disorders in aging. This view would also be consistent with the findings of another study in the same sample showing that the health and lifestyle predictors of conversion from normal cognition to MCI or any-MCD are very similar for these two clinical groups.

This study had some limitations as well as a number of strengths. The sample was limited to young-old participants in a relatively narrow age group and therefore may not apply to younger or older cohorts. The number of participants with a diagnosis was small which may have limited the power of the analyses and only broad cortical measures were investigated which could have obscured more subtle effects. Statistics were not corrected for multiple comparisons and while individual findings may not have survived stringent correction, the pattern of results across different structures suggests that they are not solely due to chance. However, the strength of this investigation was in the inclusion of a large, community-based sam- 
ple of generally healthy, well-educated individuals, the use of a wide number of predictors which have been shown in other studies to be theoretically relevant to cognitive impairment in aging, and clinical diagnoses occurring earlier in the progression to dementia and which are therefore very useful in early screening and interventions.

In summary, this study identified a limited number of absolute brain volume correlates of mild cognitive disorder which were predominantly located in the medial temporal lobe. However, in this sample stronger associations were found between mild cognitive disorders and measures of asymmetry, rather than volume in the structures investigated, suggesting that relative change in

\section{REFERENCES}

Akiyama, H., Harrop, R., McGeer, P. L., Peppard, R., and McGeer, E. G. (1989). Crossed cerebellar and uncrossed basal ganglia and thalamic diaschisis in Alzheimer's disease. Neurology 39, 541-548.

American Psychiatric Association (1994). Diagnostic and Statistical Manual of Mental Disorders (DSM-IV). Washington: APA.

Anstey, K. J., Cherbuin, N., Christensen, H., Burns, R., Reglade-Meslin, C., Salim, A., Kumar, R., Jorm, A. F., and Sachdev, P. (2008a). Follow-up of mild cognitive impairment and related disorders over 4 years in adults in their sixties: the PATH Through Life Study. Dement. Geriatr. Cogn. Disord. 26, 226-233.

Anstey, K. J., Lipnicki, D. M., and Low, L. F. (2008b). Cholesterol as a risk factor for dementia and cognitive decline: a systematic review of prospective studies with meta-analysis. Am. J. Geriatr. Psychiatry 16, 343-354.

Anstey, K. J., and Maller, J. J. (2003). The role of volumetric MRI in understanding mild cognitive impairment and similar classifications. Aging Ment. Health 7, 238-250.

Apostolova, L. G., Thompson, P. M., Green, A. E., Hwang, K. S., Zoumalan, C., Jack, C. R. Jr., Harvey, D. J., Petersen, R. C., Thal, L. J., Aisen, P. S., Toga, A. W., Cummings, J. L., and Decarli, C. S. (2010). 3D comparison of low, intermediate, and advanced hippocampal atrophy in MCI. Hum. Brain Mapp. 31, 786-797.

Barnes, J., Scahill, R. I., Schott, J. M., Frost, C., Rossor, M. N., and Fox, N. C. (2005). Does Alzheimer's disease affect hippocampal asymmetry? Evidence from a cross-sectional and longitudinal volumetric MRI study. Dement. Geriatr. Cogn. Disord. 19, 338-344.

Barnes, J., Whitwell, J. L., Frost, C., Josephs, K. A., Rossor, M., and Fox, N. C. (2006). Measurements of the amygdala and hippocampus in differences in cognitive abilities: the

volume between left and right structures might be particularly predictive of pathological changes associated with MCI and any MCD.

\section{ACKNOWLEDGMENTS}

The authors are grateful to Anthony Jorm, Helen Christensen, Patricia Jacomb, Karen Maxwell, Andrew Janke, and the path interviewers. The study was supported by NHMRC of Australia Grant No. 973302, 179805, 157125, grants from the NCI National Facility, the Australian Rotary Health Research Fund and the Australian Brewers Foundation. Nicolas Cherbuin and Kaarin Anstey are funded by NHMRC Research Fellowship No. 471501 and 366756.

mediating role of health state and health habits. Intelligence 32, 7-23.

pathologically confirmed Alzheime disease and frontotemporal lobar degeneration. Arch. Neurol. 63, 1434-1439.

Crook, T., Bartus, R., Ferris, S. H., Whitehouse, P., Cohen, G., and Gershon, S. (1986). Age-associated memory impairment: Proposed diagnostic criteria and measures of clinical change. Report of a National Institute of Mental Health Working Group. Dev. Neuropsychol. 2, 261-276.

Desikan, R. S., Fischl, B., Cabral, H. J., Kemper, T. L., Guttmann, C. R., Blacker, D., Hyman, B. T., Albert, M. S., and Killiany, R. J. (2008). MRI measures of temporoparietal regions show differential rates of atrophy during prodromal AD. Neurology 71, 819-825.

Fischl, B., Salat, D. H., Busa, E., Albert, M., Dieterich, M., Haselgrove, C., van der Kouwe, A., Killiany, R., Kennedy, D., Klaveness, S., Montillo, A., Makris, N., Rosen, B., and Dale, A. M. (2002). Whole brain segmentation: automated labeling of neuroanatomical structures in the human brain. Neuron 33, 341-355.

Fischl, B., Salat, D. H., van der Kouwe, A. J., Makris, N., Segonne, F., Quinn, B. T., and Dale, A. M. (2004). Sequenceindependent segmentation of magnetic resonance images. Neuroimage $23,69-84$.

Giannakopoulos, P., Kovari, E., Herrmann, F. R., Hof, P. R., and Bouras, C. (2009). Interhemispheric distribution of Alzheimer disease and vascular pathology in brain aging. Stroke 40, 983-986.

Jeong, Y., Song, Y. M., Chung, P. W., Kim, E. J., Kang, S. J., Kim, J. M., Cho, S. S., Kim, S. E., Byun, H. S., and Na, D. L. (2005). Correlation of ventricular asymmetry with metabolic asymmetry in frontotemporal dementia. $J$. Neuroradiol. 32, 247-254.

Jorm, A. F., Anstey, K. J., Christensen, H., and Rodgers, B. (2004). Gender
Kral, V. A. (1962). Senescent forgetfulness: benign and malignant. Can. Med. Assoc. J. 86, 257-260.

Kumar, R., Dear, K. B., Christensen, H. Ilschner, S., Jorm, A. F., Meslin, C. Rosenman, S. J., and Sachdev, P. S. (2005). Prevalence of mild cognitive impairment in 60- to 64-year-old community-dwelling individuals: the personality and total health through life 60+ Study. Dement. Geriatr. Cogn. Disord. 19, 67-74.

Kumar, R., Parslow, R. A., Jorm, A. F., Rosenman, S. J., Maller, J., Meslin, C., Anstey, K. J., Christensen, H., and Sachdev, P. S. (2006). Clinical and neuroimaging correlates of mild cognitive impairment in a middle-aged community sample: the personality and total health through life $60+$ study. Dement. Geriatr. Cogn. Disord. 21, 44-50.

Morris, J. C. (1993). The Clinical Dementia Rating (CDR): current version and scoring rules. Neurology 43, 2412-2414.

Onbas, O., Dane, S., Kantarci, M., Koplay, M., Alper, F., and Okur, A. (2007). Clinical importance of asymmetry and handedness differences in common carotid artery intima-media thickness. Int. J. Neurosci. 117, 433-441.

Oosterman, J. M., Vogels, R. L., van Harten, B., Gouw, A. A., Scheltens, P., Poggesi, A., Weinstein, H. C., and Scherder, E. J. (2008). The role of white matter hyperintensities and medial temporal lobe atrophy in age-related executive dysfunctioning. Brain Cogn 68, 128-133.

Ott, B. R., Heindel, W. C., Tan, Z., and Noto, R. B. (2000). Lateralized cortical perfusion in women with Alzheimer's disease. J. Gend. Specif. Med. 3, 29-35. C., Ivnik, R. J., Tangalos, E. G., and Kokmen, E. (1999). Mild cognitive impairment: clinical characterization
Petersen, R. C., Smith, G. E., Waring, S. and outcome. Arch. Neurol. 56, 303-308.

Raji, C. A., Becker, J. T., Tsopelas, N. D., Price, J. C., Mathis, C. A., Saxton, J. A., Lopresti, B. J., Hoge, J. A., Ziolko, S. K., DeKosky, S. T., and Klunk, W. E. (2008). Characterizing regional correlation, laterality and symmetry of amyloid deposition in mild cognitive impairment and Alzheimer's disease with Pittsburgh Compound B. J. Neurosci. Methods 172, 277-282.

Segonne, F., Dale, A. M., Busa, E., Glessner, M., Salat, D., Hahn, H. K., and Fischl, B. (2004). A hybrid approach to the skull stripping problem in MRI. Neuroimage 22, 1060-1075.

Shi, F., Liu, B., Zhou, Y., Yu, C., and Jiang, T. (2009). Hippocampal volume and asymmetry in mild cognitive impairment and Alzheimer's disease: Metaanalyses of MRI studies. Hippocampus 19, 1055-1064.

Smith, M. E. (2005). Bilateral hippocampal volume reduction in adults with post-traumatic stress disorder: a meta-analysis of structural MRI studies. Hippocampus 15, 798-807.

Thompson, P. M., Hayashi, K. M., Dutton, R. A., Chiang, M. C., Leow, A. D., Sowell, E. R., De Zubicaray, G., Becker, J. T., Lopez, O. L., Aizenstein, H. J., and Toga, A. W. (2007). Tracking Alzheimer's disease. Ann. N. Y. Acad. Sci. 1097, 183-214.

Trollor, J. N., Sachdev, P. S., Haindl, W., Brodaty, H., Wen, W., and Walker, B. M. (2005). Regional cerebral blood flow deficits in mild Alzheimer's disease using high resolution single photon emission computerized tomography. Psychiatry Clin. Neurosci. 59, 280-290.

van den Heuvel, D. M., ten Dam, V. H., de Craen, A. J., Admiraal-Behloul, F., Olofsen, H., Bollen, E. L., Jolles, J., Murray, H. M., Blauw, G. J., Westendorp, R. G., and van Buchem, M. A. (2006). Increase in periventricular white matter hyperintensities parallels decline in mental processing speed in a non-demented elderly 
population. J. Neurol. Neurosurg. Psychiatr. 77, 149-153.

Wen, W., Sachdev, P. S., Chen, X., and Anstey, K. (2006). Gray matter reduction is correlated with white matter hyperintensity volume: a voxel-based morphometric study in a large epidemiological sample. Neuroimage 29, 1031-1039.

Yoshita, M., Fletcher, E., Harvey, D., Ortega, M., Martinez, O., Mungas,
D. M., Reed, B. R., and DeCarli, C. S. (2006). Extent and distribution of white matter hyperintensities in normal aging, MCI, and AD. Neurology 67, 2192-2198.

Conflict of Interest Statement: The authors declare that the research was conducted in the absence of any commercial or financial relationships that could be construed as a potential conflict of interest.
Received: 12 January 2010; paper pending published: 03 March 2010; accepted: 07 April 2010; published online: 11 May 2010.

Citation: Cherbuin N, Réglade-Meslin C, Kumar R, Sachdev P and Anstey KJ (2010) Mild cognitive disorders are associated with different patterns of brain asymmetry than normal aging: the PATH through life study. Front. Psychiatry 1:11. doi: 10.3389/ fpsyt.2010.00011
This article was submitted to Frontiers in Neurodegeneration, a specialty of Frontiers in Psychiatry.

Copyright $\odot 2010$ Cherbuin, RégladeMeslin, Kumar, Sachdev and Anstey. This is an open-access article subject to an exclusive license agreement between the authors and the Frontiers Research Foundation, which permits unrestricted use, distribution, and reproduction in any medium, provided the original authors and source are credited. 